\title{
Potencialidades pedagógicas de los entornos de simulación, desde la perspectiva de la cognición situada
}

Germán Amaya Franky*

Artículo recibido: 27-06-2007 y aprobado: 20-10-2009

Pedagogical potentialities of the simulation, surroundings from the perspective of the located cognition

Resumen: El presente artículo muestra los resultados de una investigación desarrollada como requisito para obtener el título de Doctor. Hace referencia a las posibilidades que presentan los entornos info-virtuales de aprendizaje, más exactamente al software de simulación, como escenarios que posibilitan la contextualización de la actividad y la construcción del conocimiento significativo. Esto es, la interacción con un evento simulado puede generar aspectos que se tornan reales dentro de un sistema de actividad, posibilitando así la construcción del conocimiento ubicado en un contexto de actividad, de situación y de interpretación sociocultural.

Palabras clave: Simuladores computarizados, actividad, situación, cognición situada.
Abstract: This paper presents the results of a research study developed as a requirement for the PhD degree. It refers to the possibilities that infovirtual environments present for learning, specifically in the field of simulation software as possible scenarios that allow the contextualization of the activity and the construction of meaningful knowledge. That is, the interaction with a simulated event which may generate issues that become real within a system of activity, allowing the construction of knowledge focused on the activity context and the socio-cultural interpretation.

Key words: Computer simulations, activity, location, situated cognition.

\footnotetext{
* Universidad de Pamplona. Correo electrónico: amayag@usal.es
} 
La mayoría de aportes teóricos que se especializan en indagar la manera como los humanos construyen el conocimiento, resaltan la necesidad de desarrollar actividades en contextos concretos que permitan a los individuos encontrarle significado a lo que aprenden. En tal sentido, los eventos reales aportan un entorno de posibilidades situacionales, de significado sociocultural y de actividad específica, que facultan la construcción de un aprendizaje significativo.

La educación tradicional ha buscado por siempre adoptar marcos teóricos y metodologías de enseñanza y aprendizaje que permitan hacer de las aulas de clase un entorno significativo para las actividades pedagógicas ${ }^{1}$. Para ello, ha construido las actividades mediante aproximaciones directas a acontecimientos y situaciones del mundo real y programaciones organizadas mediante el empleo de las posibilidades estructuradoras de los textos escritos.

De la misma forma, como proyecto pedagógico en contextos de clausura institucional, surgen tecnologías ${ }^{2}$ artefactuales, teorías generales y sistemas tecnológicos, que han pretendido dar respuesta a dicha problemática. Sin embargo, se trata de contextos que, en mayor o menor medida, consiguen superar parcialmente la descontextua-

1 En palabras de Wenger: "la práctica es el proceso por el cual podemos experimentar el mundo y nuestro compromiso con él como algo significativo" (Wenger, E., 2001, p. 75).

2 Sancho define la tecnología como "el conjunto de saberes que nos permite intervenir en el mundo, como el conjunto de herramientas físicas o artefactuales, psíquicas o simbólicas y sociales $u$ organizativas" (Sancho, J., 1994, p.7). lización ${ }^{3}$ que origina todo aprendizaje formal institucionalizado.

La construcción de estas actividades desde el punto de vista del aprendizaje significativo, constituye un verdadero reto pedagógico, porque la difícil tarea de generar eventos realistas y significativos en el interior de las aulas de clase, termina siendo un objetivo y una fuente de dificultad para el proyecto de la comprensión significativa del mundo dentro de contextos institucionalizados de aprendizaje.

$\mathrm{Al}$ respecto y gracias a los adelantos en materia de Nuevas Tecnologías de la Información y la Comunicación (NTIC), florecen grandes y positivas expectativas, porque los entornos virtuales de aprendizaje permiten simular eventos reales que pueden ser un aporte metodológico a las descontextualizadas actividades educativas dentro de la educación institucionalizada.

Estos entornos de simulación, que se hallan inscritos bajo el nombre de simuladores computarizados, pueden ser una alternativa metodológica de solución a la descontextualización del aprendizaje. Cabe resaltar que, por simulación se entiende "toda la teoría relacionada con el proceso en el cual se sustituyen las situaciones reales por otras creadas artificialmente", pudiendo representar en el modelo la apariencia, la estructura, la organización de sus componentes y la dinámica del sistema. (González, V., 1990, p. 284). Por consiguiente, la simulación computarizada es la representación digital de un sistema

3 Al hacer referencia a actividades pedagógicas descontextualizadas, se hace alusión a actividades sueltas de su contexto de aplicación y uso. 
real, expresada mediante otro sistema manipulable que puede ser natural, artificial o imaginario.

Desde otro ángulo, en el plano teórico emergen planteamientos que resaltan el contexto situacional y el proceso dinámico de la actividad como ejes en la construcción del conocimiento. Un ejemplo de estos marcos teóricos es la cognición situada. Este marco de inteligibilidad resalta el hecho que "el conocimiento está situado, siendo en parte un producto de actividad, del contexto y de la cultura en la cual se desarrolla y se utiliza" (Brown, J., Collins, A. \& Duguid, P., 1989, pp. 33-42).

La cognición situada demanda que las actividades deben ser lo más realistas posibles, para que las interacciones y demás elementos del contexto, permitan a los educandos encontrar significado a lo que se aprende. Al respecto, una vez más, se resalta el carácter fundamental de los entornos reales en la construcción del conocimiento. Estos hechos exhortan a buscar alternativas de solución que permitan generar eventos realistas y contextos de actividad situada en espacios como las aulas de clase o entornos digitales de educación institucionalizada.

Variadas investigaciones (Akpan, J., 2001; Orlansky, J. \& String, J., 1977; Wolfe, J., 1975) han demostrado que las experiencias, vivencias y manipulación de materiales reales dentro del contexto metodológico, proporcionan a los aprendices la oportunidad de contextualizar el aprendizaje. Sin embargo, no es posible en todo momento proporcionar a los aprendices dichos entornos, ya sea por su costo o difícil accesibilidad. Entonces, se hace necesario identificar las posibilidades pedagógicas de las tecnologías info-virtuales como posibles espacios de simulación de la realidad, y como posible respuesta a la problemática de la descontextualización que se genera en todo aprendizaje institucionalizado. Es decir, es pertinente verificar si estos entornos de realidad simulada por ordenador, pueden en algún momento ser tan significativos para la construcción del conocimiento como los entornos de realidad que se generan dentro de un laboratorio verdadero. Al respecto, se genera el siguiente cuestionamiento:

La instrucción basada en una metodología del conocimiento situado dentro de una realidad simulada ipuede ser tan significativa para la construcción del conocimiento, como la instrucción que desde la misma metodología se sitúa en contextos de laboratorio reales?

Este problema es el que otorga origen a la presente investigación, así, encontrar la respuesta es el objetivo primordial.

\section{Diseño de la investigación}

Esta tesis doctoral ha permitido abarcar dos grandes retos: El primero consistió en establecer un marco teórico con el fin de interpretar los entornos de simulación computarizada como posibles instrumentos del método pedagógico en la contextualización del aprendizaje y por consiguiente, en la construcción del pensamiento consciente. Como segundo, se planteó una actividad empírica que permitió demostrar la veracidad de los elementos teóricos establecidos anteriormente.

Como marco de inteligibilidad para el análisis teórico, se han tomado los aportes de la teoría de la cognición situada, ya que ésta resalta la contextualización como elemento primordial 
en la construcción del conocimiento. Este análisis permitió, desde el punto de vista teórico, divisar los entornos computarizados de simulación como espacios que verdaderamente posibilitan la contextualización en el proceso de enseñanza y aprendizaje.

Para la fase experimental se han tenido presentes los lineamientos del diseño investigativo cuasiexperimental. Se ha elegido un modelo con pruebaposprueba y grupos intactos. Este diseño se caracteriza por presentar dos grupos, uno experimental y otro control. Por otro lado, a ambos grupos se les administra una preprueba y una posprueba. Su esquema representativo sería el siguiente:

\begin{tabular}{cccc}
\hline Grupos & Medida de la pre-prueba & Tratamiento & Medida de la pos-prueba \\
\hline Experimental & $\mathrm{O}_{1}$ & $\mathrm{X}$ & $\mathrm{O}_{2}$ \\
\hline Control & $\mathrm{O}_{1}$ & - & $\mathrm{O}_{2}$ \\
\hline
\end{tabular}

Tabla1. Diseño cuasiexperimental con pre-prueba y pos-prueba.

Según la naturaleza de los datos, la presente investigación se enmarca dentro de un enfoque dominante, donde predomina el modelo cuantitativo, aunque algunos datos requieren de un análisis cualitativo.

Así mismo, se ha seleccionado una muestra conformada por 50 estudiantes, de los cuales 32 integraron el grupo experimental y 18 el grupo control. El primero usó el simulador como instrumento para la contextualización de las actividades pedagógicas y el segundo, con la misma finalidad, usó el contexto de laboratorio con material real.

Hay que destacar que este trabajo no se ha centrado en comprobar la efectividad de una metodología pedagógica, sino las potencialidades de los simuladores como instrumentos del método en la construcción del conocimiento desde un marco de inteligibilidad establecido. Por otro lado, es también pertinente resaltar que la finalidad de esta investigación no está centrada en proponer una forma de trabajo para una asignatura específica, sino en la contextualización de un aprendizaje en general.
Sin embargo, se hace necesario abordar un eje de conocimiento que permita diseñar las actividades instructivas para la fase experimental, por tal razón y para este cometido, se considera adecuado incursionar en una temática que más que memorización, exige la lógica y la aplicación de un aprendizaje consciente, significativo y acorde con el contexto situacional. Con este objetivo se ha seleccionado para la presente investigación, la especialidad de física dentro del currículo de la educación secundaria de Colombia; más concretamente el referido al comportamiento de la corriente eléctrica dentro de los circuitos eléctricos de corriente continua (ley de Ohm).

Los objetivos e hipótesis propuestos en el presente estudio, se pueden resumir así:

\section{Objetivos}

Evaluar y comparar la eficacia de la simulación computarizada frente a la eficacia de las actividades desarrolladas con material real, como instrumentos del método en la construcción y aplicación del conocimiento conceptual, procedi- 
mental, y como método que posibilita mejorar la actitud de los aprendices hacia una asignatura específica. Así mismo, se pretende medir la transferencia de dicho aprendizaje a otros contextos y la posibilidad de retención de dicho aprendizaje a mediano plazo.

\section{Hipótesis:}

Los estudiantes que reciben instrucción basada en cognición situada, usando el entorno de simulación computarizado como sustituto de la realidad, presentan el mismo nivel de resultados, en cuanto a construcción y reconstrucción del aprendizaje conceptual y procedimental, a la transferencia del aprendizaje a otros contextos, y a la mejora de la actitud de los educandos frente a una asignatura específica.

\section{La cognición situada}

El concepto de cognición situada ha sido tratado por diversos autores, que concurren en un principio particular de definiciones que giran en torno a una misma concepción: el carácter situacional que se hereda del legado cultural, la interacción que emerge dentro de la actividad, la influencia de la situación en la toma de decisiones y la interpretación, son algunas de las características más comunes en toda concepción sobre cognición situada.

Retomando algunas de las concepciones que, de la cognición situada, se presentan, sobresalen, en primer lugar, autores como Brown, J., Collins, A. \& Duguid, P., quienes desaprueban las actividades educativas y la adquisición de conceptos lejos de los ambientes en los cuales el aprendizaje tiene pertenencia, es decir, lejos de donde se aprende y se utiliza. Por tal razón, manifiestan que "el conocimiento está situado, siendo en parte un producto de la actividad, del contexto y de la cultura en la cual se desarrolla y se utiliza" (Brown, J., 1989, p. 33-42).

Otro de los aportes a la concepción sobre la cognición situada, se debe a dos autores: Lave, J. \& Wenger, E., quienes han dedicado gran parte de sus investigaciones al análisis de este tema, y en sus contribuciones es claro dilucidar las ideas de Vygotsky, en donde se toma la cultura como agente acumulador de "sistemas simbólicos socialmente creados, donde se encuentran los organizadores de la experiencia" (Rodríguez, M. \& García, M., 2003, 136-144).

Para estos autores [Lave y Wenger], el aprendizaje situado es un aspecto inseparable e integral de cualquier práctica social. La noción de aprendizaje situado indica precisamente el carácter contextualizado del aprendizaje que no se reduce a las nociones convencionales de aprendizaje in situ o aprendizaje haciendo, sino a la participación del aprendiz en una comunidad de práctica o sea en un contexto cultural, social de relaciones, en donde obtiene los saberes necesarios para transformarla y transformarse (Ibídem).

Otro aporte lo constituyen las ideas de Clancey, W. (1995), quien al igual que Lave, J., ha realizado contribuciones significativas al carácter de las situaciones y el entorno en la aprehensión del conocimiento en contextos situacionales. Desde esta postura, el aprendizaje situado es visto como una actividad natural y cotidiana del conocimiento humano. Se caracteriza porque la construcción del conocimiento se genera de forma dinámica mediante la interacción con la situación. Así, la cognición situada es el 
"concepto de la actividad dentro de una matriz de formas sociales que motivan los pensamientos, actos, acciones y decisiones". Por consiguiente, la acción se sitúa en el papel de los miembros de una comunidad.

\section{Contextos de simulación}

La simulación se define como un "acontecimiento estructurado que involucra relaciones causales entre el elemento y evento que representa una situación del mundo real" (Duchastel, 1990, en Martí, E., 1992). Desde otro aporte, Delval, J. (1986) define la simulación como la reproducción de una situación o un fenómeno que se presenta generalmente simplificado y que a su vez, permite la manipulación de sus variables intervinientes. En tal sentido, las simulaciones deben constituir un "modelo de situación o de fenómeno, en el que aparecen los aspectos que se consideran importantes para nuestro propósito, despreciando así los que son secundarios o accesorios" (Delval, J., 1986, p. 154).

Por tanto, un entorno o modelo de simulación pretende representar el desarrollo de la realidad en forma parcial, en otras palabras, procura representar en parte el funcionamiento de un sistema real. En tal caso, la simulación computarizada es la representación digital de una parte de la realidad, la cual está expresada mediante un sistema info-virtual manipulable que puede ser natural, artificial o imaginario. Desde los conceptos anteriormente esbozados, es pertinente unificar dos palabras que se tornan indispensables en la interpretación del término simulación.

a. Sistema: Es "una colección o conjunto de elementos o normas que ordenados cumplen un fin particular"(Martínez, S. \& Requena, A., 1986, p. 34). En palabras de Shannon, R. (1988), un sistema es un conjunto de objetos o ideas que están interrelacionados entre sí como una unidad para la consecución de un fin lógico. El autor también lo define como la porción del universo que será objeto de la simulación. Así, las anteriores definiciones hacen entrever que la característica esencial de un sistema, es la interrelación existente entre cada una de sus partes con relación a ciertas reglas enmarcadas dentro de un objetivo. En consecuencia, un sistema puede realizar una función que no es realizable por sus componentes en forma individual.

b. Modelo: Es un objeto o evento que puede ser empleado por un individuo para indagar y responder por aspectos de su interés con relación al objeto o evento real. En palabras de Law, A. \& Kelton, W. (1991) Un objeto $\mathrm{X}$ es un modelo del objeto $\mathrm{Y}$ para el observador Z, si Z puede emplear a X para responder cuestiones que le interesan acerca de Y. En tal sentido, un modelo de simulación es "el conjunto de hipótesis acerca del funcionamiento del sistema expresado como relaciones matemáticas y/o lógicas entre los elementos del sistema" (García, C. \& Villatoro, F., 2004, cap. 1).

\section{Potencialidades pedagógicas de los entornos de simulación}

Desde una revisión de los antecedentes que enmarcan esta investigación, es evidente percibir que los entornos de simulación son recursos que presentan diversos aportes al sector de la educación. Sin embargo, la efectividad de las herramientas utilizadas en el proceso de enseñanza y aprendizaje no depende 
sólo de los artefactos, sino que existen muchos otros factores que influyen directamente, tales como: estilos, ritmos, preferencias, capacidades, estrategias de aprendizaje y antecedentes historiales del aprendiz con relación a los conocimientos previos. Por consiguiente, "el aprendizaje depende esencialmente del ser que aprende, no de forma directa de lo que desea o se propone el que enseña" (Gimeno, J. y Pérez, A., 1992).

Se hace necesario reconocer en los contextos un elemento potenciador de las motivaciones que conllevan a generar aprendizaje. El entorno educativo adecuadamente prediseñado, potencia las posibilidades de los individuos para la construcción del conocimiento, porque el factor pedagógico de los entornos depende en gran medida de la metodología empleada; entendida ésta como "la ciencia que estudia los métodos, técnicas, procedimientos y medios dirigidos a la enseñanza de una disciplina dada". Así, las tecnologías deben ser vistas como herramientas para instrumentar los métodos y no como métodos propiamente dichos (Arias, L., 2004).

El uso de elementos tecnológicos como los simuladores computarizados, debe ser visto como un recurso que media y faculta a los aprendices en la construcción del conocimiento. Las simulaciones computarizadas deben ser vistas como recursos técnicos de orden material, que le facilitan al educando la interacción, estudio, y/o modelación de la realidad o de una parte de ésta. Desde esta perspectiva, "los ordenadores personales constituyen medios técnicos de especial significación para el contexto metodológico actual" (Arias, L., 2004) y no elementos pedagógicos por sí so- los. En definitiva, el valor pedagógico y didáctico de los entornos de simulación, es proporcionado por el contexto metodológico en el que se explotan sus cualidades.

Si la simulación es vista como un recurso que facilita la ejecución de metodologías educativas y, por consiguiente, de procedimientos de aprendizaje ¿por qué no se deben clasificar como metodología propiamente dicha?

Para que la simulación se pueda clasificar como una metodología, tendría que ser por sí sola un elemento suficiente para penetrar en la esencia de la realidad, y esto no es así, pues la modelación de un evento real no es un hecho suficiente para que se produzcan actividades de interacción significativa. Desde aquí se hace necesario el empleo de procedimientos, que en forma secuencial ayuden a la interpretación de esa realidad.

Por otra parte, y desde un enfoque sociocultural del aprendizaje, las simulaciones por sí solas no pueden generar eventos e interacciones sociales o sistemas colaborativos; es cierto que sí generan un entorno común de significados, pero dependiendo de un proceso metodológico que induce a la reflexión. En consecuencia, se deben reconocer las simulaciones como elementos que coaccionan con la metodología en el proceso de enseñanza y aprendizaje, porque las simulaciones son instrumentos del método que corresponden a una parte de las actividades que comprenden un diseño metodológico y, no son por sí solas metodología de enseñanza y aprendizaje. 


\section{Desarrollo de la experimentación, aplicación de las pruebas y resultados}

Con el fin de posibilitar la actividad empírica, se ha hecho necesario abordar una temática instruccional y una especificidad en cuanto al ámbito del aprendizaje a evaluar dentro de los logros instruccionales. Por tanto, las condiciones de la investigación exigen que se deba tener presente una temática instruccional, que más que la memorización, sugiera la aplicación consciente del conocimiento. Al respecto, y revisando las ciencias, más específicamente la física, se decide trabajar con la construcción de circuitos eléctricos y la aplicación de la ley de Ohm.
Por otra parte, se planean las actividades pedagógicas que guiarán la instrucción, bajo los lineamientos de la cognición situada. Estos aspectos han permitido plantear una serie de actividades que sugieren a los aprendices generar interacciones significativas con el contexto de situación, de actividad y sociocultural.

Los instrumentos utilizados para recopilar la información, han sido pruebas elaboradas ad hoc y pruebas adaptadas, utilizadas en otras investigaciones. Para el análisis estadístico se han aplicado pruebas paramétricas y no paramétricas según el caso. Dicho análisis en la comparación de las dos metodologías, han presentado los siguientes resultados.

\section{Diferencias presentadas}

\begin{tabular}{ll}
\hline \multicolumn{1}{c}{ Objetivo de evaluación } & Grupo experimental vs. grupo control \\
\hline Aprendizaje en conceptos & No hay diferencias significativas \\
Aprendizaje en procedimientos & No hay diferencias significativas \\
Actitud hacia la asignatura & No hay diferencias significativas \\
Transferencia del aprendizaje & No hay diferencias significativas \\
Retención del aprendizaje en conceptos & Diferencias en favor del grupo experimental \\
Retención de la actitud & No hay diferencias significativas \\
\hline
\end{tabular}

Tabla 2. Diferencias presentadas en los grupos experimental y control.

\begin{tabular}{lccccc}
\hline Total prueba & $\mathbf{N}$ & Media & $\begin{array}{c}\text { Desviación } \\
\text { típica }\end{array}$ & $\mathbf{- t}$ & $\mathbf{- p}$ \\
\hline Experimental & 32 & 14,29 & 3,778 & 2,569 & 0 \\
\hline Control & 18 & 11,33 & 3,955 & & \\
\hline
\end{tabular}

Tabla 3. Resultados para la comparación de grupos en la prueba de conceptos, dos meses de concluida la instrucción, total de la prueba. 


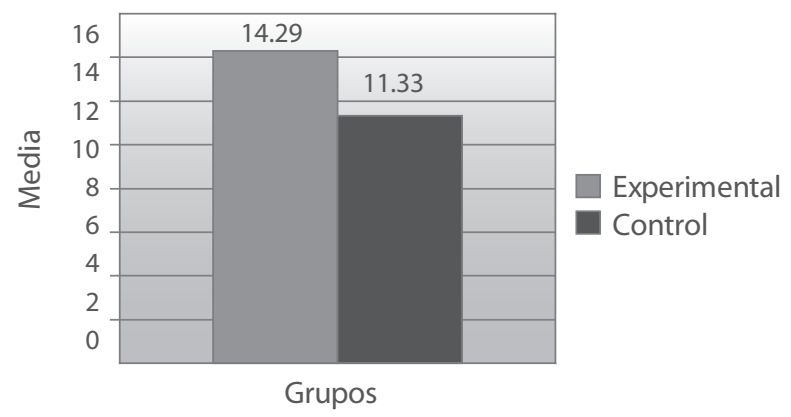

Gráfico 1. Resultados de la comparación de grupos en la prueba de conceptos, dos meses después de concluida la instrucción.

\section{Conclusiones}

Los resultados arrojados luego de aplicar los análisis estadísticos, han permitido establecer las siguientes conclusiones.

1. La cognición situada permite el ancla de las actividades y posibilita la construcción consciente y significativa del conocimiento.

2. El simulador permite magnificar el aprendizaje conceptual, cuando el factor tiempo interviene en las relaciones causales que posibilitan su aprehensión

3. En la construcción del aprendizaje de procedimientos, un laboratorio real puede ser reemplazado, al menos en algunos casos, por un entorno de simulación computarizada, generando la misma conceptualización.

\section{Referencias bibliográficas}

Arias, L. (2004). La simulación computarizada como procedimiento metodológico en la enseñanza y el aprendizaje de la Electrónica. Extraído el 26 de Marzo, 2005, desde http://www.monografias.com/trabajos13/simucom/ simucom.shtml

Akpan, J. (2000). Using a Computer Simulation Before Dissection to Help Students Learn Anatomy [Versión electrónica]. Jl. of Computers in $\mathrm{Ma}$ -
4. Los entornos de simulación pueden reemplazar los contextos de laboratorio real, en el momento que se desee mejorar la transferencia (descontextualización) del aprendizaje.

5. El aprendizaje mediado por entornos de simulación, posibilita el aprendizaje a mediano plazo en mayor proporción que el aprendizaje mediado por entornos de laboratorio real.

Dados los resultados de la investigación, que concuerdan con los resultados de otras investigaciones, se puede afirmar que los entornos de simulación posibilitan a los individuos un contexto significativo de aprehensión y uso de las herramientas conceptuales.

thematicsandScienceTeaching, 19(3), 297-313, desde http://www.aace.org/ dl/files/jcmst/jcmst-19-03-297.pdf

Akpan, J. (2001). Issues Associated with Inserting Computer Simulations into Biology Instruction: A Review of the Literature [Versión electrónica]. Electronic journal of science education, 5 (3). http://unr.edu/homepage/ crowther/ejse/akpan.html 
Brown, J.; Collins, A. y Duguid, P. (1989). Situated Cognition and the culture of learning, Educational Researcher, 18(1), 33-42.

Castells, M. (1999). La era de la información. Economía, sociedad y cultura, vol. 3. Fin de milenio. Madrid: Alianza.

Castells, M. (2001). Materiales para una teoría preliminar sobre la sociedad de redes. Revista de Educación, número extraordinario, 41-58.

Chaiklin, S. y Lave, J. (2001). Estudiar las prácticas. Argentina: Amorrortu.

Clancey, W. (1995). A tutorial on situated learning. Proceedings of the International Conference on Computers and Education (Taiwan) Charlottesville, 49-70. Extraído el 18 de Abril, 2004, de http://www.cogprints.ecs.soton. ac.uk/archive/00000323/00/139. htm.

Delval, J. (1986). Niños y máquinas. Los ordenadores y la educación. Madrid: Alianza.

García, C. \& Villatoro, F. (2004). Técnicas de simulación mediante el método montecarlo. Extraído el 7 de Enero, 2005, de http://polaris.lcc.uma. es/ villa/mmtc/tema14.pdf.
Gimeno, J. y Pérez, A. (1992). Comprender y transformar la enseñanza. Madrid: Morata.

González Castro, V. (1990). Teoría y práctica de los medios de enseñanza. La Habana: Pueblo y Educación.

Lave, J. y Wenger, E. (1991). Situated Learnig Legitimate Peripheral Participation. USA: Cambridge University Press.

Law, A. y Kelton W. (1991). Simulation Modeling \& Analysis ( $\left.2^{\mathrm{a} e d}\right)$. New York: McGraw-Hill.

Martí, E. (1992). Cuadernos de educación. Aprender con ordenadores en la escuela. Barcelona: Ice-Horsori.

Martínez, S. y Requena, A. (1986). Dinámica de sistemas. Simulación por ordenador. Madrid: Alianza.

Rodríguez, M. y García, M. (2003). El aprendizaje como problema actual de la educación. Revista Cubana de Investigación, 20 (2), 136-144.

Sancho, J. (1994). Para una tecnología educativa. Barcelona: Horsori.

Shannon, R. (1988). Simulación de sistemas. Diseño, desarrollo e implementación. México D. F.: Trillas.

Wenger, E. (2001). Comunidades de práctica. Barcelona: Paidós. 